lustitia Socialis. Revista Arbitrada de Ciencias Jurídicas.

Año V. Vol. V. N³. Edición Especial. 2020-III:

Universidad Regional Autónoma de los Andes

Hecho el depósito de Ley: FA2016000064 ISSN: 2542-3371

FUNDACIÓN KOINONIA (F.K). Santa Ana de Coro, Venezuela

Danilo Rafael Andrade-Santamaria; Manuel Ricardo Velázquez;

Jocelyne Leonela Calles-Quinaluiza; José Luis Castro-Fiallos

http://dx.doi.org/10.35381/racji.v5i3.1064

\title{
Violencia intrafamiliar versus acoso laboral a los trabajadores del GAD Parroquial de Shell
}

\section{Domestic violence and workplace harassment of Shell Parish GAD workers}

\author{
Danilo Rafael Andrade-Santamaria \\ up.daniloandrade@uniandes.edu.ec \\ Universidad Regional Autónoma de los Andes, Puyo \\ Ecuador \\ https://orcid.org/0000-0002-1405-5710 \\ Manuel Ricardo Velázquez \\ up.manuelricardo@uniandes.edu.ec \\ Universidad Regional Autónoma de los Andes, Puyo \\ Ecuador \\ https://orcid.org/0000-0001-5357-8197 \\ Jocelyne Leonela Calles-Quinaluiza \\ leonelacalles327@hotmail.com \\ Universidad Regional Autónoma de los Andes, Puyo \\ Ecuador \\ José Luis Castro-Fiallos \\ joseluiscastrol@hotmail.com \\ Universidad Regional Autónoma de los Andes, Puyo \\ Ecuador
}

Recibido: 31 de octubre de 2020

Revisado: 10 de octubre de 2020

Aprobado: 05 de diciembre de 2020

Publicado: 10 de diciembre de 2020 
lustitia Socialis. Revista Arbitrada de Ciencias Jurídicas.

Año V. Vol. V. N³. Edición Especial. 2020-III:

Universidad Regional Autónoma de los Andes

Hecho el depósito de Ley: FA2016000064

ISSN: 2542-3371

FUNDACIÓN KOINONIA (F.K). Santa Ana de Coro, Venezuela

Danilo Rafael Andrade-Santamaria; Manuel Ricardo Velázquez;

Jocelyne Leonela Calles-Quinaluiza; José Luis Castro-Fiallos

\title{
RESUMEN
}

El objetivo general de la presente investigación es difundir mediante charlas a los trabajadores del GAD Parroquial de Shell acerca de la violencia intrafamiliar y la figura del acoso laboral. Metodológicamente fue de tipo descriptiva, la muestra poblacional estuvo conformada por 20 trabajadores. Se ha determinado que el $65 \%$ de los trabajadores desconocen que el Código de Trabajo reconoce la figura del acoso laboral, el $75 \%$ de los trabajadores si han denunciado violencia intrafamiliar. La violencia en cualquiera de sus formas es, sin lugar a duda, la expresión más cruda del ejercicio del poder: el hombre sobre la mujer, el adulto de ambos sexos sobre los niños y niñas, el rico sobre el pobre y en general el fuerte sobre el débil, las sociedades humanas han tratado de regular, mediante la promulgación de leyes, el ejercicio arbitrario de la violencia, fundamentalmente con el fin de proteger a los más vulnerables.

Descriptores: Violencia doméstica; abuso de menores; acoso. (Palabras tomadas del Tesauro UNESCO).

\begin{abstract}
The general objective of this research is to disseminate through talks to the workers of the Shell Parish GAD about domestic violence and the figure of workplace bullying. Methodologically it was descriptive, the population sample consisted of 20 workers. It has been determined that $65 \%$ of workers are unaware that the Labor Code recognizes the figure of workplace harassment, $75 \%$ of workers have reported domestic violence. Violence in any of its forms is, without a doubt, the crudest expression of the exercise of power: the man over the woman, the adult of both sexes over the boys and girls, the rich over the poor and in general the strong. on the weak, human societies have tried to regulate, through the promulgation of laws, the arbitrary exercise of violence, fundamentally in order to protect the most vulnerable.
\end{abstract}

Descriptors: Domestic violence; child abuse; bullying. (Words taken from the UNESCO Thesaurus). 
lustitia Socialis. Revista Arbitrada de Ciencias Jurídicas.

Año V. Vol. V. N³. Edición Especial. 2020-III:

Universidad Regional Autónoma de los Andes

Hecho el depósito de Ley: FA2016000064

ISSN: 2542-3371

FUNDACIÓN KOINONIA (F.K). Santa Ana de Coro, Venezuela

Danilo Rafael Andrade-Santamaria; Manuel Ricardo Velázquez;

Jocelyne Leonela Calles-Quinaluiza; José Luis Castro-Fiallos

\section{INTRODUCCIÓN}

La violencia intrafamiliar se define como un conjunto de actos que atenta contra la integridad física, psicológica y económica de algún miembro de la familia, normalmente las víctimas de la violencia intrafamiliar son niños y mujeres, pero en la actualidad también se ha incrementado violencia intrafamiliar hacia los hombres. Existen diferentes tipos de violencia, tanto en la familia, así como también en la materia laboral y uno de ellos es la figura llamada acoso laboral. Los trabajadores, la mayoría de las ocasiones han preferido callar ante el maltrato ejercido dentro de sus trabajos y hogares, por miedo a perder sus trabajos y perder su hogar en el cual y han encontrado estabilidad.

El gran nivel de aumento de la violencia intrafamiliar y laboral, que día a día ha ido incrementándose dentro de nuestro entorno, nos ha llevado a enfocarnos dentro del GAD Parroquial de Shell, ha sido notorio que desde hace años atrás y en la actualidad se han vulnerado sus derechos, por eso a través de este proyecto establecer los lineamientos básicos que nos conducirán a un estudio más acabado del problema, así nos permita conocer de mejor manera las causas, consecuencias reales.

La incidencia de violencia intrafamiliar y acoso laboral en el GAD Parroquial de Shell, cantón y provincia de Pastaza afecta el derecho a la integridad personal y laboral, determinado en la Constitución de la República del Ecuador, puesto que genera daños psicológicos, físicos e inestabilidad emocional y mal ambiente laboral.

Por lo anterior, el problema de investigación es: ¿Cuáles son las razones por las que son víctimas de violencia intrafamiliar y laboral los trabajadores del GAD Parroquial de Shell? El objetivo general de la presente investigación es difundir mediante charlas a los trabajadores del GAD Parroquial de Shell acerca de la violencia intrafamiliar y la figura del acoso laboral. 
lustitia Socialis. Revista Arbitrada de Ciencias Jurídicas.

Año V. Vol. V. N³. Edición Especial. 2020-III:

Universidad Regional Autónoma de los Andes

Hecho el depósito de Ley: FA2016000064

ISSN: 2542-3371

FUNDACIÓN KOINONIA (F.K). Santa Ana de Coro, Venezuela

Danilo Rafael Andrade-Santamaria; Manuel Ricardo Velázquez;

Jocelyne Leonela Calles-Quinaluiza; José Luis Castro-Fiallos

\section{Los objetivos específicos que responden al objetivo general son los siguientes:}

a) Fundamentar social y jurídicamente, las causas y consecuencias de la violencia intrafamiliar y acoso laboral.

b) Promover información a través de charlas motivacionales y capacitación por medio de la entrega de trípticos, sobre la violencia intrafamiliar y laboral en la GAD Parroquial de Shell.

c) Elaborar una propuesta para disminuir el acoso laboral y la violencia intrafamiliar en el GAD Parroquial de Shell.

- Determinar la incidencia de la violencia intrafamiliar acoso laboral en los trabajadores del GAD Parroquial de Shell.

\section{Referencial teórico}

\section{Violencia intrafamiliar}

Es necesario precisar que la violencia intrafamiliar y laboral ha ido tomando fuerza con el pasar del tiempo, por ello hemos tomado como referencia EI GAD Parroquia de Shell, en donde se ha evidenciado la existencia de personas que han sido afectadas, siendo necesario dar a conocer a la sociedad de nuestra localidad, para que de esta forma se tome conciencia.

Es de suma importancia estudiar este fenómeno que se da en cualquier acto que afecta al supervivencia de la víctima, la violencia familiar ocurre dentro y fuera del domicilio y como consecuencia se puede evidenciar destrucciones matrimoniales y el quebramiento familiar; la violencia laboral se ejerce hacia la personas que tiene un vínculo de dependencia laboral consiste en un acto o una omisión de abuso de poder que daña el autoestima, salud integridad, libertad y seguridad del trabajador e impide su desarrollo y atenta contra la igualdad. Es necesario que los trabajadores del GAD parroquia de Shell conozcan acerca de sus derechos, a la integridad personal y un buen ambiente laboral y así prever este tipo de problema que afecta a la familia y al trabajo. 
lustitia Socialis. Revista Arbitrada de Ciencias Jurídicas.

Año V. Vol. V. N³. Edición Especial. 2020-III:

Universidad Regional Autónoma de los Andes

Hecho el depósito de Ley: FA2016000064

ISSN: 2542-3371

FUNDACIÓN KOINONIA (F.K). Santa Ana de Coro, Venezuela

Danilo Rafael Andrade-Santamaria; Manuel Ricardo Velázquez;

Jocelyne Leonela Calles-Quinaluiza; José Luis Castro-Fiallos

La violencia es una realidad compleja que ha caracterizado la historia de la humanidad y está presente en todas las relaciones sociales su reconocimiento y visibilidad depende de los criterios de normalidad aceptada por las sociedades de acuerdo a cánones históricamente definidos en el Ecuador los índices de violencia, en general, han aumentado notoriamente en la última década como consecuencia de los procesos de desintegración social desatados por la transición de modelo de desarrollo y la crisis nacional. La violencia no se limita a las agresiones físicas, y tiene raíces en la historia y la cultura de la sociedad y en el lugar que nosotras ocupamos en ella y se cree que este problema es un asunto exclusivamente de sus miembros, en consecuencia, nadie está autorizado a intervenir (Aguilar-Freire \& Rodríguez-Hidalgo, 2018).

La violencia familiar es un término aplicado al maltrato físico y emocional de una persona por parte de alguien que está en estrecha relación con la víctima el término incluye la violencia en la pareja (hombres o mujeres), el maltrato físico, el abandono del niño, el abuso sexual del niño, el maltrato del anciano y muchos casos de agresión sexual (DíazCárdenas, 2015), pudiendose originar en cualquier país, sin importar sexo, etnia, religioso posición socio económica, aunque las definiciones varían según la cultura, la violencia intrafamiliar representa un problema de salud pública, debido a las muertes, heridas y consecuencias psicológicas que provoca.

El daño físico y emocional puede representar impedimentos crónicos o de por vida para muchas víctimas a la violencia familiar puede ocasionar angustia, depresión, comportamiento autodestructivo y, en casos extremos, el suicidio las víctimas, a menudo, se convierten en agresores o participan en relaciones violentas más tarde (PuenteMartínez, et al., 2016).

La violencia intrafamiliar es una realidad compleja que ha caracterizado la historia de la humanidad y está presente en todas las relaciones sociales su reconocimiento y visibilidad depende de los criterios de "normalidad" aceptada por las sociedades de acuerdo a cánones históricamente definidos en el Ecuador los índices de violencia, en 
lustitia Socialis. Revista Arbitrada de Ciencias Jurídicas.

Año V. Vol. V. N³. Edición Especial. 2020-III:

Universidad Regional Autónoma de los Andes

Hecho el depósito de Ley: FA2016000064

ISSN: 2542-3371

FUNDACIÓN KOINONIA (F.K). Santa Ana de Coro, Venezuela

Danilo Rafael Andrade-Santamaria; Manuel Ricardo Velázquez;

Jocelyne Leonela Calles-Quinaluiza; José Luis Castro-Fiallos

general, han aumentado notoriamente en la última década como consecuencia de los procesos de desintegración social desatados por la transición de modelo de desarrollo y la crisis nacional, así como por la emergencia por COVID-19 (Montero-Medina, et al., 2020).

Es así como desde tiempos muy remotos ha existido la violencia contra la mujer y la familia en general, la mujer ha tenido que soportar el maltrato continuo de sus parejas, ya sea por proteger a sus hijos o por sentirse desprotegida económicamente. El maltrato no solamente ha sido físico sino psicológico el cual afecta a su autoestima en primer lugar, lo que la hace un blanco fácil para la violencia (Castillo-Saavedra, et al., 2018).

La violencia intrafamiliar también expresa un abuso de poder por parte de un miembro de la familia sobre otro este abuso incluye maltrato físico, psicológico o de cualquier otro tipo se considera que existe este problema en un hogar si este se vuelve repetitivo, más no por un hecho aislado, la víctima de violencia intrafamiliar es cualquier persona considerada cónyuge del agresor o que haya convivido de alguna manera con él (Moralde-la-Rubia \& López-Rosales, 2013).

El fenómeno de la violencia doméstica o familiar se ha convertido en las últimas décadas en un asunto de máximo interés institucional y social atendiendo, principalmente, a razones como su elevada incidencia y la gravedad de las consecuencias que de él se derivan, el conocimiento real de la incidencia de este tipo de violencia se ve principalmente obstaculizado por el ocultamiento social al que tradicionalmente se ha asociado el sufrimiento de malos tratos por parte de una figura del ámbito familiar.

La violencia doméstica, familiar o intrafamiliar: comprende todos aquellos actos donde el empleo de la fuerza física, incluso matonaje, acoso e intimidación- en el seno de un hogar proviene de por lo menos un miembro de la familia en contra algún otro familiar.

Sobre la violencia física se plantea teniendo en cuenta que la agresividad es un componente biológico de muchos animales, incluyendo al ser humano. Lo que lo 
lustitia Socialis. Revista Arbitrada de Ciencias Jurídicas.

Año V. Vol. V. N³. Edición Especial. 2020-III:

Universidad Regional Autónoma de los Andes

Hecho el depósito de Ley: FA2016000064

ISSN: 2542-3371

FUNDACIÓN KOINONIA (F.K). Santa Ana de Coro, Venezuela

Danilo Rafael Andrade-Santamaria; Manuel Ricardo Velázquez;

Jocelyne Leonela Calles-Quinaluiza; José Luis Castro-Fiallos

diferencia de los animales es que puede agregar otros componentes, como la conciencia y la voluntad de hacer daño, transformándola en violencia física.

Por otro lado, la violencia sexual hace referencia al acto de coacción hacia una persona con el objeto de que lleve a cabo una determinada conducta sexual. Por extensión, se consideran también como ejemplos de violencia sexual los comentarios o insinuaciones sexuales no deseados, o las acciones para comercializar o utilizar de cualquier otro modo la sexualidad de una persona mediante coacción por parte de otra persona, independientemente de la relación de ésta con la víctima, en cualquier ámbito, incluidos el hogar y el lugar de trabajo.

\section{Acoso laboral}

El acoso laboral puede obedecer a motivos laborales o extralaborales, pero se ha de realizar en el lugar de trabajo o si se realiza en otro lugar, sea como consecuencia de la relación laboral. En la definición de acoso laboral podemos observar dos formas distintas de manifestarse o realizarse, que influyen a la hora de realizar su detección, evaluación, afrontamiento, tratamiento y erradicación.

La figura del Acoso Laboral o en términos inglés "Moobing", es un inconveniente que se ha desarrollado en el ambiente laboral, causado por el irrespeto, ofensas, malos tratos entre el empleador y su trabajador o entre compañeros de trabajo, ocasionando la afectación psicológica de quien recibe estos malos tratos, además que lo previsto en las normativas ecuatorianas, protegen al trabajador pero en la práctica no existe una adecuada socialización de los derechos de la clase trabajadora, que consideramos como la parte débil y de fácil ataque por parte de sus superiores o compañeros acoso de forma horizontal (Rosales-Quichimbo, et al., 2019).

El Acoso Laboral induce a comportamientos inadecuados e irrespeto por parte del acosador hacia su víctima (Lanata-Fuenzalida, 2018), trayendo consigo un clima inestable, incierto, e inseguro de trabajo, y por qué no decirlo también de desconfianza, 
lustitia Socialis. Revista Arbitrada de Ciencias Jurídicas.

Año V. Vol. V. N³. Edición Especial. 2020-III:

Universidad Regional Autónoma de los Andes

Hecho el depósito de Ley: FA2016000064 ISSN: 2542-3371

FUNDACIÓN KOINONIA (F.K). Santa Ana de Coro, Venezuela

Danilo Rafael Andrade-Santamaria; Manuel Ricardo Velázquez; Jocelyne Leonela Calles-Quinaluiza; José Luis Castro-Fiallos

convergen diversos tipos de problemas (físicos, psicológicos, intelectuales, credibilidad). Así mismo trae como consecuencia el desprestigio, el buen desempeño del empleado, además, la institución se ve involucrada en este tipo de figura jurídica por lo que esto genera comentarios negativos efectuados por todas aquellas víctimas que fueron obligadas a abandonar su trabajo por no aceptar propuestas o intenciones ajenas al trabajo y que van en contra de la moral y la voluntad de cada ser humano, por otro lado, Piñuel, (2004) lo define como:

El continuo y deliberado maltrato verbal y modal que recibe un trabajador por parte de otro u otros, que se comportan con él cruelmente con vistas a lograr su aniquilación o destrucción psicológica y a obtener su salida de la organización a través de diferentes procedimientos.

Debe entenderse por acoso laboral todo comportamiento, atentatorio a la dignidad de la persona, ejercido de forma reiterada, y potencialmente lesivo, cometido en el lugar de trabajo o en cualquier momento en contra de una de las partes de la relación laboral o entre trabajadores, que tenga como resultado para la persona afectada su menoscabo, maltrato, humillación, o bien que amenace o perjudique su situación laboral en este sentido, (Soares-Cugnier \& Silva, 2016), hacen el llamado para prevenir este tipo de acción en los ambientes laborales, con la finalidad de generar un clima organizacional óptimo para el desempeño del talento humano, situación corroborada en el estudio de (Álava-Tagle, et al., 2020), quienes señalan que el trabajador al sufrir acoso laboral, baja su autoestima y esto influye en su rendimiento.

Puede señalarse que el mobbing es una forma de estrés laboral conducente a cuaros depresivos (Harasemiuc \& Díaz-Bernal, 2013), por lo tanto, tiene su origen en las relaciones entre las personas que se generan entre las personas. Algunas de las variables que pueden originar el acoso son:

a) La organización del trabajo: a la víctima del mobbing se le empiezan a atribuir tareas para que realice mal su trabajo. Esto se consigue cuando, por un lado, se le atribuyen cometidos que tienen una sobrecarga cuantitativa. Es decir, el 
lustitia Socialis. Revista Arbitrada de Ciencias Jurídicas.

Año V. Vol. V. N³. Edición Especial. 2020-III:

Universidad Regional Autónoma de los Andes

Hecho el depósito de Ley: FA2016000064

ISSN: 2542-3371

FUNDACIÓN KOINONIA (F.K). Santa Ana de Coro, Venezuela

Danilo Rafael Andrade-Santamaria; Manuel Ricardo Velázquez;

Jocelyne Leonela Calles-Quinaluiza; José Luis Castro-Fiallos

trabajador tendrá mucha demanda de trabajo, pero poco control sobre él. Por otro lado, tendrá un déficit cualitativo, se verá obligado a realizar un trabajo que se caracteriza por ser aburrido, inútil y repetitivo. Todo esto origina un estrés que puede ser el caldo de cultivo idóneo para que empiecen a aparecer conflictos y culmine en un tipo de maltrato psicológico y mobbing.

b) La dirección: los jefes autoritarios, aquellos que buscan optimizar y tener los mejores resultados, pero, sin tener en cuenta el factor humano pueden causar también conflictos que desemboquen finalmente en acoso laboral.

c) Las tareas: cuando una persona realiza siempre las mismas tareas, la monotonía y la repetición constante puede provocar malestar y conflictos entre los miembros del equipo. Si el trabajo no supone un reto para el empleado o no le permita crecer a nivel laboral o personal pueden acabar causando presión, frustración que en muchas ocasiones deriva en que ese trabajador canalice su malestar con alguna forma de acoso hacia otro trabajador.

d) Desprestigio ante el resto de los trabajadores: el acoso laboral también puede aparecer ante actividades que tienen el objetivo de desacreditar la reputación laboral o personal. Para conseguirlo el agresor maldecirá, calumniará, inventará rumores, cotilleos o enfermedades de la víctima. También lo ridiculizará mediante la imitación de gestos, posturas o de su voz, se burlará de su vida privada, de su nacionalidad, de sus orígenes o intentará llevarlo al extremo, incluso atribuyéndole enfermedades mentales.

e) Desprestigio laboral: los acosadores también pueden realizar acciones y actividades de acoso que pretendan reducir la ocupación de la víctima y su empleabilidad al desprestigiarle laboralmente. Para conseguirlo el responsable no le asignará ningún trabajo, se le asignan quehaceres inútiles o absurdos, inferiores a su capacidad o competencias profesionales o, por el contrario, le imponen tareas que exigen tener más experiencia y conocimientos a sus competencias. 
lustitia Socialis. Revista Arbitrada de Ciencias Jurídicas.

Año V. Vol. V. N³. Edición Especial. 2020-III:

Universidad Regional Autónoma de los Andes

Hecho el depósito de Ley: FA2016000064

ISSN: 2542-3371

FUNDACIÓN KOINONIA (F.K). Santa Ana de Coro, Venezuela

Danilo Rafael Andrade-Santamaria; Manuel Ricardo Velázquez;

Jocelyne Leonela Calles-Quinaluiza; José Luis Castro-Fiallos

En este sentido, se hace necesario que las organizaciones laborales, cuenten con protocolos para evitar el acoso laboral en sus instalaciones, aunado de tratar jurídica y psicológicamente al trabajador que lo padezca, así como al causante, por cuanto podría ser un reflejo de sus relaciones familiares, generándose un circulo de violencia social que va en detrimento de la justicia y paz colectiva.

Por otro lado, el Estado, los consejos provinciales, las municipalidades y demás personas jurídicas de derecho público tienen la calidad de empleadores respecto de los obreros de las obras públicas nacionales o locales. Se entiende por tales obras no sólo las construcciones, sino, también el mantenimiento de las mismas y, en general, la realización de todo trabajo material relacionado con la prestación de servicio público, aun cuando a los obreros se les hubiere extendido nombramiento y cualquiera que fuere la forma o período de pago, siendo necesario que, en estos organismos públicos, se profundice en la prevención del acoso laboral que implícitamente coopera en la reducción de la violencia intrafamiliar.

\section{MÉTODO}

Se empleó primordialmente la modalidad cuantitativa, con una tipología descriptiva con diseño no experimental de campo, la muestra poblacional estuvo conformada por 20 trabajadores del GAD Parroquial Rural de Shell, ubicada en el cantón Mera, provincia Pastaza - Ecuador. Para la recolección de datos, se empleó como técnica la encuesta, como instrumento se aplicó un cuestionario de 7 preguntas y alternativas de respuestas diversas (Ver Anexo 1), siendo validado a través del juicio de expertos y se calculó el coeficiente Alfa de Cronbach con resultado de 0,87 siendo confiable para su aplicación. Una vez aplicado el cuestionario y recopilada la información, esta fue analizada mediante estadística descriptiva, obteniéndose porcentajes de participación que fueron representados a través de los gráficos diseñados, de ese modo, se interpretó percepción de la población estudiada en relación a las variables de investigación. 
lustitia Socialis. Revista Arbitrada de Ciencias Jurídicas.

Año V. Vol. V. №3. Edición Especial. 2020-III:

Universidad Regional Autónoma de los Andes

Hecho el depósito de Ley: FA2016000064

ISSN: 2542-3371

FUNDACIÓN KOINONIA (F.K). Santa Ana de Coro, Venezuela

Danilo Rafael Andrade-Santamaria; Manuel Ricardo Velázquez;

Jocelyne Leonela Calles-Quinaluiza; José Luis Castro-Fiallos

\section{RESULTADOS}

Los principales resultados obtenidos de la aplicación de la encuesta (Anexo 1) se presentan a continuación, de tal manera que se enuncia la pregunta realizada, los resultados obtenidos de la tabulación de estas y el análisis que se hace de ellas:

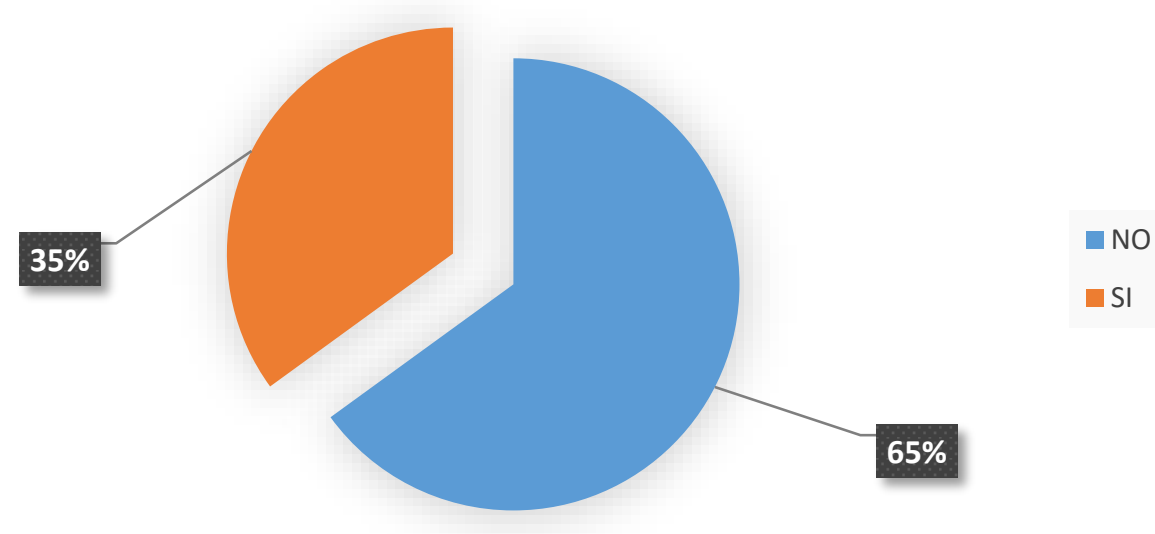

Gráfico 1. ¿Sabe usted que el Código de Trabajo reconoce la figura del acoso laboral?

Se ha determinado que el 65\% de los trabajadores desconocen que el Código de Trabajo reconoce la figura del acoso laboral y el $35 \%$ de los trabajadores no conocen.

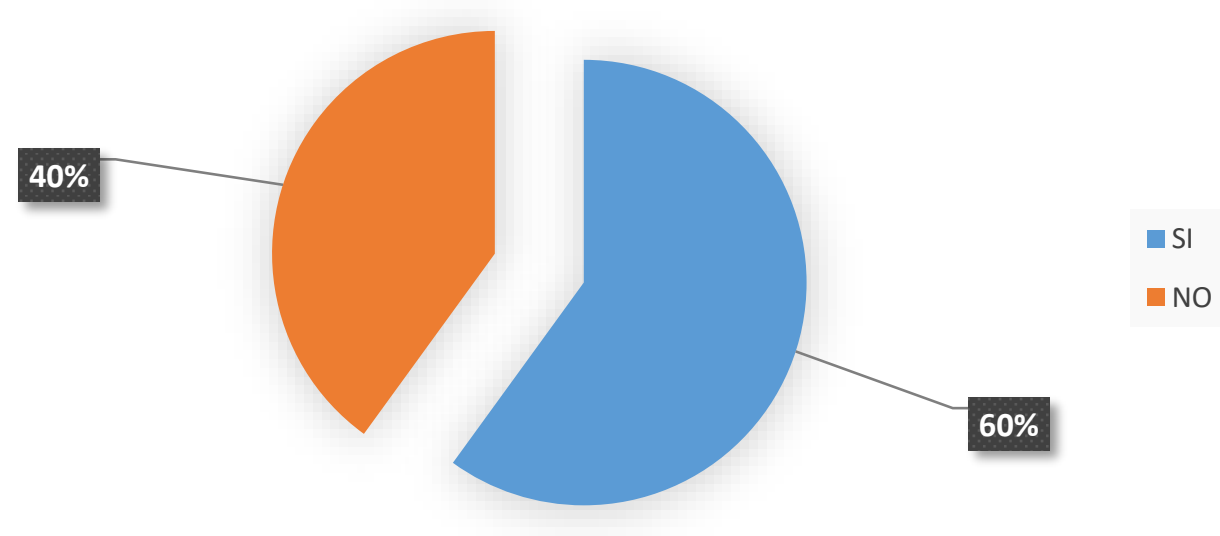

Gráfico 2. ¿Ha sufrido alguna vez acoso laboral? 
lustitia Socialis. Revista Arbitrada de Ciencias Jurídicas.

Año V. Vol. V. №3. Edición Especial. 2020-III:

Universidad Regional Autónoma de los Andes

Hecho el depósito de Ley: FA2016000064

ISSN: 2542-3371

FUNDACIÓN KOINONIA (F.K). Santa Ana de Coro, Venezuela

Danilo Rafael Andrade-Santamaria; Manuel Ricardo Velázquez;

Jocelyne Leonela Calles-Quinaluiza; José Luis Castro-Fiallos

El $60 \%$ de los trabajadores ha sufrido alguna vez acoso laboral y el $40 \%$ de los trabajadores no lo han sufrido, lo cual es significativo para la presente investigación.

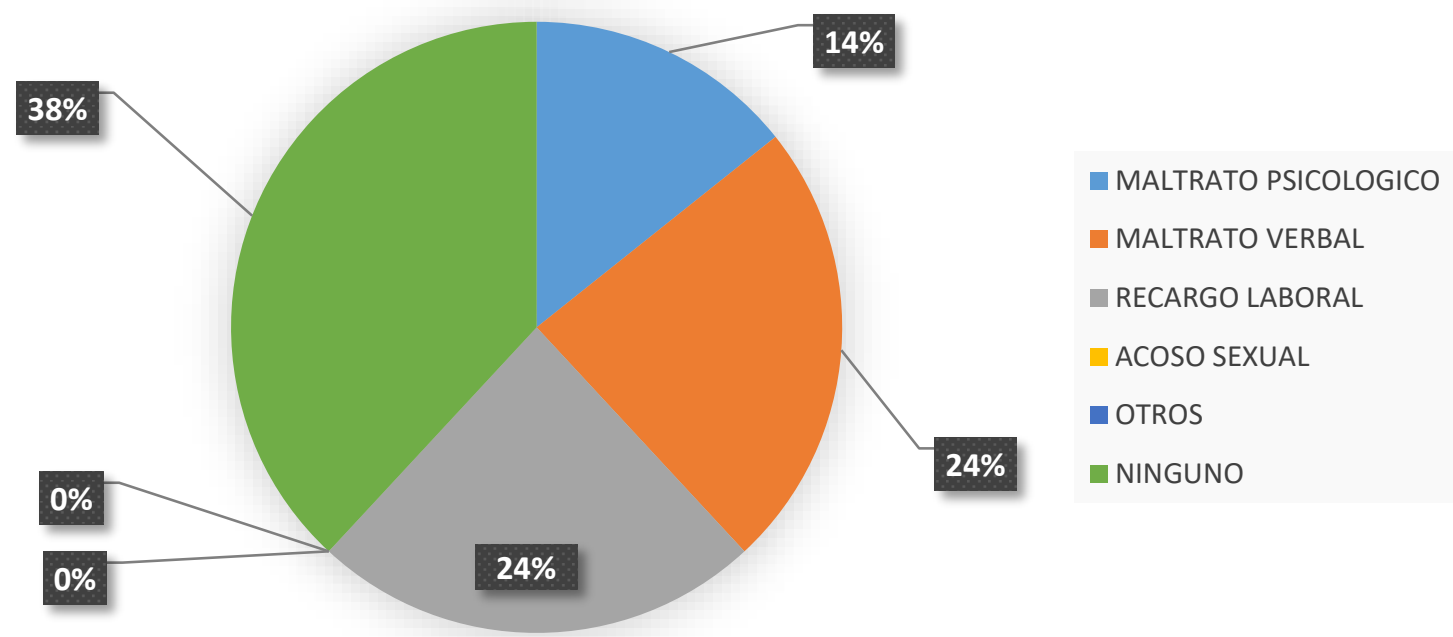

Gráfico 3. ¿De qué atipo de acoso laboral ha sido víctima o testigo?

Se ha determinado que el $62 \%$ de los trabajadores ha sufrido algún tipo de acoso laboral y el $38 \%$ de los trabajadores no lo han sufrido, y los tipos más influyentes son el maltrato verbal y el recargo laboral.

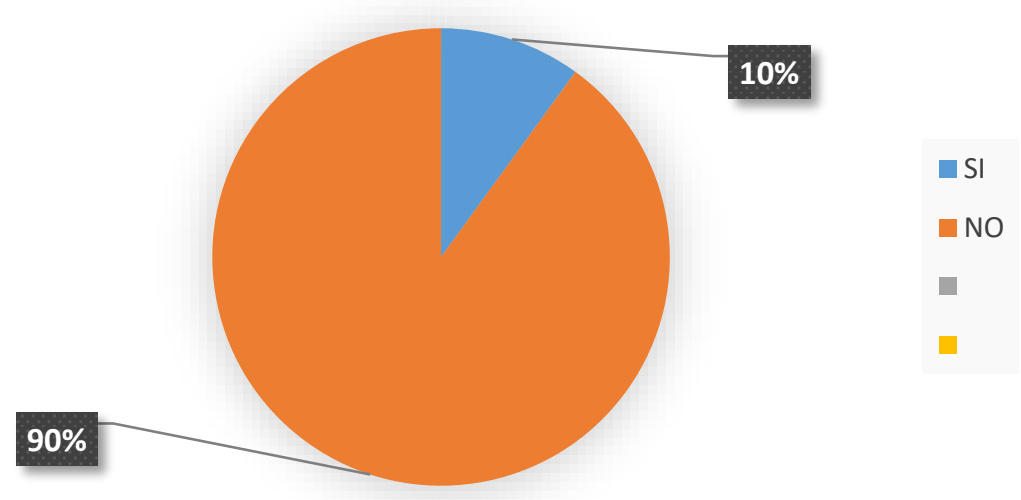

Gráfico 4. ¿Ha denunciado alguna vez acoso laboral? 
lustitia Socialis. Revista Arbitrada de Ciencias Jurídicas.

Año V. Vol. V. №3. Edición Especial. 2020-III:

Universidad Regional Autónoma de los Andes

Hecho el depósito de Ley: FA2016000064 ISSN: 2542-3371

FUNDACIÓN KOINONIA (F.K). Santa Ana de Coro, Venezuela

Danilo Rafael Andrade-Santamaria; Manuel Ricardo Velázquez;

Jocelyne Leonela Calles-Quinaluiza; José Luis Castro-Fiallos

El $90 \%$ de los trabajadores no ha denunciado acoso laboral y el $10 \%$ de los trabajadores si lo han hecho.

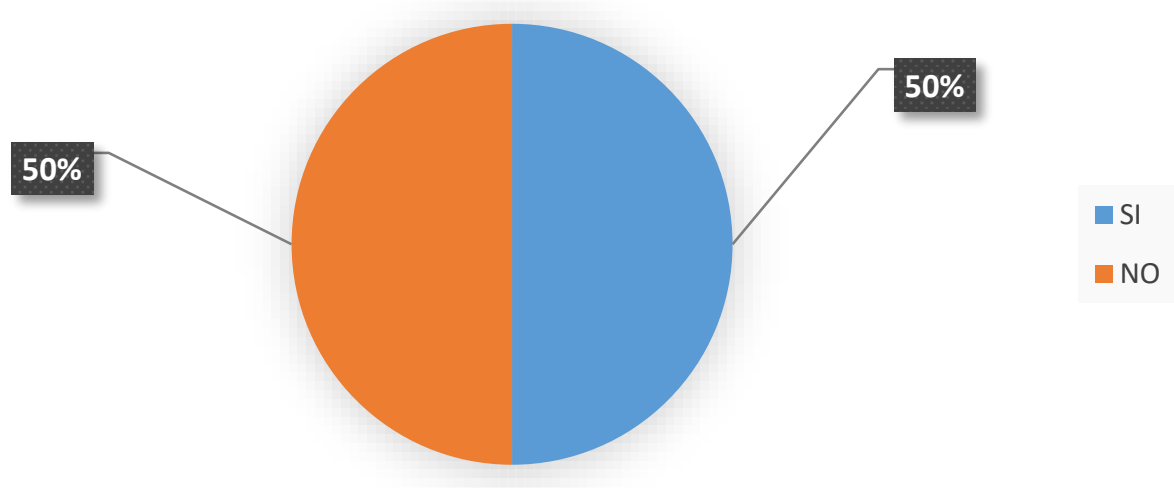

Gráfico 5. Violencia intrafamiliar sufrida.

De la pregunta anterior se ha determinado que el $50 \%$ de los trabajadores si han sufrido alguna vez violencia intrafamiliar y el $50 \%$ de los trabajadores si no han sufrido este tipo de violencia.

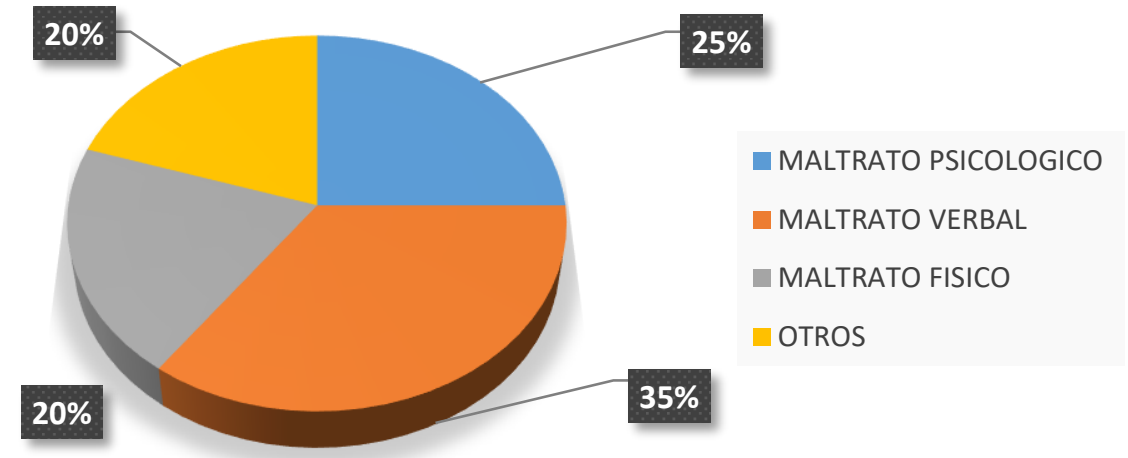

Gráfico 6. ¿De qué tipo de violencia intrafamiliar ha sido víctima o testigo? 
lustitia Socialis. Revista Arbitrada de Ciencias Jurídicas.

Año V. Vol. V. №3. Edición Especial. 2020-III:

Universidad Regional Autónoma de los Andes

Hecho el depósito de Ley: FA2016000064

ISSN: 2542-3371

FUNDACIÓN KOINONIA (F.K). Santa Ana de Coro, Venezuela

Danilo Rafael Andrade-Santamaria; Manuel Ricardo Velázquez;

Jocelyne Leonela Calles-Quinaluiza; José Luis Castro-Fiallos

Los resultados presentados en el gráfico, evidencian una presencia de maltrato psicológico en $25 \%$, maltrato verbal en $35 \%$, maltrato físico $20 \%$ y el $20 \%$ restante considera que ha sufrido otro tipo de maltrato.

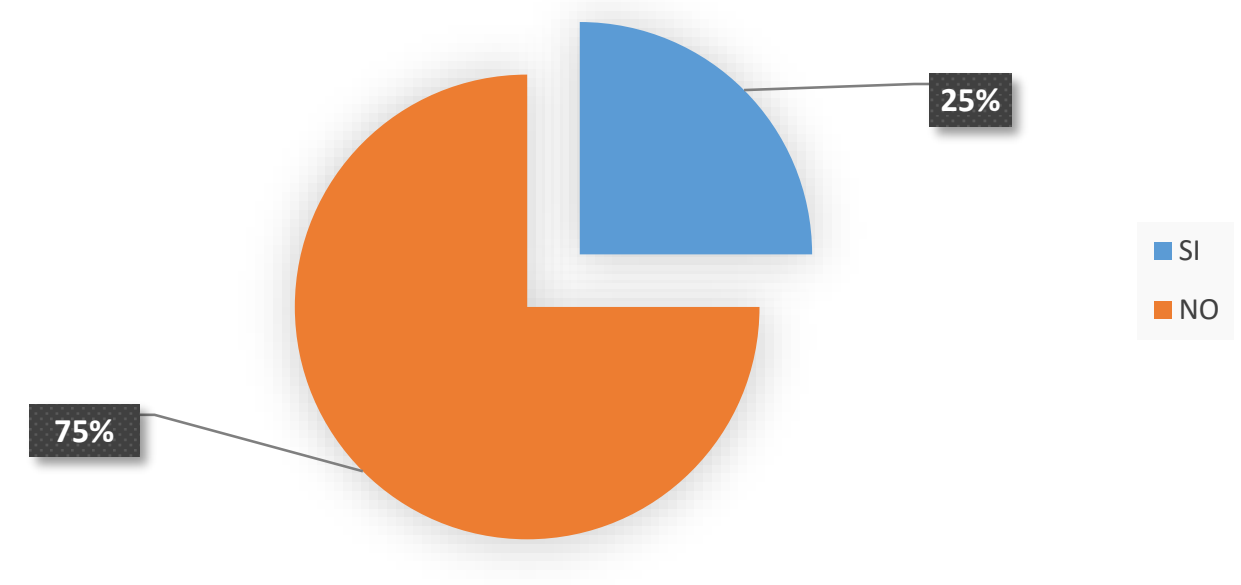

Gráfico 7. ¿Ha denunciado violencia intrafamiliar?

De las respuestas se ha determinado que el $75 \%$ de los trabajadores si han denunciado violencia intrafamiliar y el $25 \%$ de los trabajadores no han denunciado violencia intrafamiliar.

\section{DISCUSIÓN}

Los factores que originan la violencia intrafamiliar pueden ser muy variados. La intolerancia es uno de esos factores o causas. Cuando las personas no son educadas para tolerar, para aceptar, terminan volcándose violentamente contra otras, requiriéndose generar una educación emocional en favor de proteger a la familia de la violencia (Moralde-la-Rubia \& López-Rosales, 2013).

De igual forma la falta de control de los impulsos es otras de las causas. Cuando no se ha tenido un buen desarrollo de vínculos afectivos, las personas no son capaces de establecerlos, por lo tanto, adoptan conductas agresivas. Otra de las causas es la 
lustitia Socialis. Revista Arbitrada de Ciencias Jurídicas.

Año V. Vol. V. N³. Edición Especial. 2020-III:

Universidad Regional Autónoma de los Andes

Hecho el depósito de Ley: FA2016000064

ISSN: 2542-3371

FUNDACIÓN KOINONIA (F.K). Santa Ana de Coro, Venezuela

Danilo Rafael Andrade-Santamaria; Manuel Ricardo Velázquez; Jocelyne Leonela Calles-Quinaluiza; José Luis Castro-Fiallos

incapacidad de resolver problemas. Hay causas como el alcohol, el consumo de drogas. Todas estas causas pueden evitarse o corregirse con la ayuda de un psicólogo.

El acoso laboral se puede dar por distintas razones, entre las que podemos destacar los celos, la envidia, no sólo centrada esta última sobre los bienes materiales de la víctima, sino sobre las cualidades personales positivas de la misma, como puede ser su inteligencia, su brillantez personal, su ecuanimidad, relacionándose con las investigaciones de (Harasemiuc \& Díaz-Bernal, 2013) y (Soares-Cugnier \& Silva, 2016), quienes hacen un llamado de prevención del acoso laboral, con la finalidad de promover relaciones interpersonales saludables en razón de consolidar un estado emocional organizacional favorecedor de un ambiente libre de violencia.

Sin embargo, es pertinente tener en cuenta que el acoso laboral, puede originarse sobre todo cuando se incorporan nuevos trabajadores jóvenes y preparados, amenazando la promoción profesional e incluso la permanencia del acosador en la organización o viceversa.

\section{PROPUESTA}

Para poder concientizar sobre las consecuencias negativas de la violencia intrafamiliar y acoso laboral se desarrollaron charlas informativas para darles a conocer las leyes que les protegen de estos tipos de violencia, a través de un sistema de capacitaciones informativas en el ámbito jurídico a los trabajadores del GAD Parroquial de Shell, con el título de "Dile no a la violencia y ponle freno al acoso laboral".

El objetivo de la propuesta es socializar con los trabajadores del GAD Parroquial de Shell, mediante charlas informativas, las causas y consecuencias legales, que produce la violencia intrafamiliar y acoso laboral, para de esta manera reducir el alto índice de estas figuras en dicha institución.

Para el desarrollo de nuestro tema, los recursos de investigación que utilizamos son: recursos humanos, documentos, equipos, transporte, recursos monetarios, materiales, 
lustitia Socialis. Revista Arbitrada de Ciencias Jurídicas.

Año V. Vol. V. N³. Edición Especial. 2020-III:

Universidad Regional Autónoma de los Andes

Hecho el depósito de Ley: FA2016000064 ISSN: 2542-3371

FUNDACIÓN KOINONIA (F.K). Santa Ana de Coro, Venezuela

Danilo Rafael Andrade-Santamaria; Manuel Ricardo Velázquez; Jocelyne Leonela Calles-Quinaluiza; José Luis Castro-Fiallos

técnicos y la realización de una encuesta. El recurso humano es indispensable para cualquier grupo social; ya que de ellos depende el manejo y funcionamiento de los demás recursos. Los documentos: que empleamos son impresiones para realizar las debidas encuestas, fotos del lugar en donde vamos a investigar en este caso en el GAD Parroquial de Shell, acceso a internet para la información sobre los antecedentes del acoso laboral y violencia intrafamiliar, también hemos vinculado los artículos de los diferentes mecanismos sancionatorios y garantistas como son: la constitución de la república del Ecuador, el Código Orgánico Integral Penal, el Código de trabajo y el Código Civil.

Los equipos, son aquellos que sirven como herramientas e instrumentos auxiliares en la coordinación de los otros recursos: software, cámara fotográfica, impresoras, grabadora, computadora, celular son los equipos que implementaremos para garantizar el desarrollo de nuestro proyecto integrador.

En el ámbito financiero utilizamos los recursos monetarios propios para el buen funcionamiento y desarrollo de nuestra investigación, en donde se llevará a cabo conforme consta en el presupuesto realizado.

El planteamiento de la propuesta se encuentra conectado directamente con el problema que se detalló en las páginas iniciales de nuestra investigación. La realidad social la cual estamos investigando constituye un enorme problema, cuya gravedad se está poniendo cada vez más en manifiesto, este tipo de comportamiento lo hemos podido observar a diario, ya que va incrementándose desmesuradamente en los hogares y en los lugares de trabajo. Considerando que el artículo 66, inciso 3 numeral a y b de la Constitución de la República del Ecuador (2008), indica que:

El derecho a la integridad personal, que incluye: a) La integridad física, psíquica, moral y sexual. b) Una vida libre de violencia en el ámbito público y privado. El Estado adoptará las medidas necesarias para prevenir, eliminar y sancionar toda forma de violencia, en especial la ejercida contra las mujeres, niñas, niños y adolescentes, personas adultas mayores, personas con discapacidad y contra toda persona en situación de desventaja o vulnerabilidad; idénticas medidas se tomarán contra la violencia, la esclavitud y la explotación sexual. 
lustitia Socialis. Revista Arbitrada de Ciencias Jurídicas.

Año V. Vol. V. N³. Edición Especial. 2020-III:

Universidad Regional Autónoma de los Andes

Hecho el depósito de Ley: FA2016000064

ISSN: 2542-3371

FUNDACIÓN KOINONIA (F.K). Santa Ana de Coro, Venezuela

Danilo Rafael Andrade-Santamaria; Manuel Ricardo Velázquez;

Jocelyne Leonela Calles-Quinaluiza; José Luis Castro-Fiallos

Existen diferentes causas por las que los trabajadores del GAD Parroquial de Shell son víctimas de violencia intrafamiliar y acoso laboral, dentro de nuestra investigación hemos podido detectar que los mismo tienen desconocimiento de la existencia de una figura legal que los protege del acoso laboral, así como también como denunciar estos tipos de violencia.

Nuestra propuesta llamada: Sistema de capacitaciones informativas en el ámbito jurídico a los trabajadores del GAD Parroquial de Shell, "Dile no a la violencia y ponle freno el acoso laboral" la misma que la realizamos mediante charlas educativas e informativas para así evitar que se aumente el índice de trabajadores que desconocen como denunciar estas figuras.

Mediante profesionales voluntarios del tema realizamos charlas para poder dar a conocer cuáles son las medidas legales que podemos tomar para dejar de ser víctimas de estas figuras en caso de serlo, así también darles a conocer cómo podemos denunciarlas ante quien y cuál es el procedimiento para seguir en estos casos.

\section{CONCLUSIONES}

El análisis obtenido con la realización de encuestas en el GAD Parroquial de Shell nos ha podido mostrar el alto porcentaje de trabajadores que desconocen como denunciar el acoso laboral y la violencia intrafamiliar.

Hemos podido determinar que las causas y consecuencias más comunes por las que no se denuncia la violencia intrafamiliar y acoso laboral se da por miedo a un superior hacer agredidas o a perder sus trabajos, así como sus familias.

La violencia se establece como una forma habitual de comunicación derivada de la intolerancia frente a una forma diferente de relación que no sea complementaria y la no aceptación de la genuina paridad en otras palabras, los episodios de violencia se desencadenarán cuando esta relación complementaria se vea amenazada, el equilibrio estable se rompa y el poder se imponga mediante el uso de la fuerza. 
lustitia Socialis. Revista Arbitrada de Ciencias Jurídicas.

Año V. Vol. V. N³. Edición Especial. 2020-III:

Universidad Regional Autónoma de los Andes

Hecho el depósito de Ley: FA2016000064 ISSN: 2542-3371

FUNDACIÓN KOINONIA (F.K). Santa Ana de Coro, Venezuela

Danilo Rafael Andrade-Santamaria; Manuel Ricardo Velázquez; Jocelyne Leonela Calles-Quinaluiza; José Luis Castro-Fiallos

La violencia en cualquiera de sus formas es, sin lugar a duda, la expresión más cruda del ejercicio del poder: el hombre sobre la mujer, el adulto de ambos sexos sobre los niños y niñas, el rico sobre el pobre y en general el fuerte sobre el débil, las sociedades humanas han tratado de regular, mediante la promulgación de leyes, el ejercicio arbitrario de la violencia, fundamentalmente con el fin de proteger a los más vulnerables.

\section{FINANCIAMIENTO}

No monetario.

\section{AGRADECIMIENTO}

A la Universidad Regional Autónoma de Los Andes, Ecuador; por motivar el desarrollo de la Investigación.

\section{REFERENCIAS CONSULTADAS}

Aguilar-Freire, D., \& Rodríguez-Hidalgo, C. (2018). El femicidio en la prensa ecuatoriana: análisis de contenido de los diarios "El Universo" y "El Comercio\&quot [Femicide in Ecuadorian press: content analyses of "El Universo" and "El Comercio"]. Estudios Sobre El Mensaje Periodístico, 24(1), 13-32. https://doi.org/10.5209/ESMP.59935

Álava-Tagle, S., Erazo-Álvarez, J., Narváez-Zurita, C., \& Trelles-Vicuña, D. (2020). Protección del derecho al trabajo frente al acoso laboral en el Ecuador [Protection of the right to work against workplace harassment in Ecuador]. IUSTITIA SOCIALIS, 5(9), 464-484.

Castillo-Saavedra, E, Bernardo-Trujillo, J, \& Medina-Reyes, M. (2018). Violencia de género y autoestima de mujeres del centro poblado Huanja - Huaraz, 2017 [Gender violence and self-esteem in women from the Huanja - Huaraz hamlet, 2017]. Horizonte Médico (Lima), 18(2), 4752. https://dx.doi.org/10.24265/horizmed.2018.v18n2.08 
lustitia Socialis. Revista Arbitrada de Ciencias Jurídicas.

Año V. Vol. V. N³. Edición Especial. 2020-III:

Universidad Regional Autónoma de los Andes

Hecho el depósito de Ley: FA2016000064 ISSN: 2542-3371

FUNDACIÓN KOINONIA (F.K). Santa Ana de Coro, Venezuela

Danilo Rafael Andrade-Santamaria; Manuel Ricardo Velázquez; Jocelyne Leonela Calles-Quinaluiza; José Luis Castro-Fiallos

Constitución de la República del Ecuador (2008). Capítulo sexto Derechos de libertad. [Chapter Six Freedom rights]. Registro Oficial 449 de 20-oct-2008. Recuperado de https://n9.cl/xo9c

Díaz-Cárdenas, S, \& Arrieta-Vergara, K, \& González-Martínez, F. (2015). Violencia intrafamiliar y factores de riesgo en mujeres afrodescendientes de la ciudad de Cartagena [Intrafamily violence and risk factors in Afro-descendant women in the city of Cartagena]. Revista Clínica de Medicina de Familia, 8(1),19-30.

Harasemiuc, V, \& Díaz-Bernal, J. (2013). Evidencia científica de la relación entre acoso laboral y depresión [Evidence based relationship between mobbing and depression]. Medicina y Seguridad del Trabajo, 59(232), 361371. https://dx.doi.org/10.4321/S0465-546X2013000300006

Lanata-Fuenzalida, R. (2018). El acoso laboral y la obligación de seguridad en el trabajo [Labor harassment and the obligation of safety at work]. Revista de derecho (Valdivia), 31(1), 105-126. https://dx.doi.org/10.4067/S0718-09502018000100105

Montero-Medina, D., Bolívar-Guayacundo, M., Aguirre-Encalada, L., \& MorenoEstupiñán, A. (2020). Violencia intrafamiliar en el marco de la emergencia sanitaria por el COVID-19 [Domestic violence in the framework of the health emergency caused by COVID-19]. CienciAméRica, 9(2), 261-267.

Moral-de-la-Rubia, J, \& López-Rosales, F. (2013). Relación de violencia en la familia de origen y machismo, con violencia en la pareja [Relationship of family-of-origin violence and machismo with couple violence]. Medicina Universitaria; 15(59):7380.

Piñuel, I. (2004). Neomanagement: Jefes tóxicos y sus víctimas. Madrid: Ed. Aguilar.

Puente-Martínez, A, Ubillos-Landa, S, Echeburúa, E, \& Páez-Rovira, D. (2016). Factores de riesgo asociados a la violencia sufrida por la mujer en la pareja: una revisión de meta-análisis y estudios recientes [Risk factors associated with the violence against women in couples: a review of meta-analyzes and recent studies]. Anales de Psicología, 32(1), 295-306. https://dx.doi.org/10.6018/analesps.32.1.189161

Rosales-Quichimbo, C, García-Muñoz, S, \& Durán-Ocampo, A. (2019). Algunas consideraciones sobre la aplicación del derecho laboral. Machala, Ecuador [Some considerations on the application of labor law. Machala, Ecuador]. Revista Universidad y Sociedad, 11(4), 106-117. 
lustitia Socialis. Revista Arbitrada de Ciencias Jurídicas.

Año V. Vol. V. №3. Edición Especial. 2020-III:

Universidad Regional Autónoma de los Andes

Hecho el depósito de Ley: FA2016000064

ISSN: 2542-3371

FUNDACIÓN KOINONIA (F.K). Santa Ana de Coro, Venezuela

Danilo Rafael Andrade-Santamaria; Manuel Ricardo Velázquez;

Jocelyne Leonela Calles-Quinaluiza; José Luis Castro-Fiallos

Soares-Cugnier, J, \& Silva, N. (2016). Acoso laboral y su prevención en organizaciones saludables [Workplace harassment and its prevention in healthy organizations]. Salud de los Trabajadores, 24(1),43-58.

C2020 por los autores. Este artículo es de acceso abierto y distribuido según los términos y condiciones de la licencia Creative Commons Atribución-NoComercial-Compartirlgual 4.0 Internacional (CC BY-NC-SA 4.0) (https://creativecommons.org/licenses/by-nc-sa/4.0/). 